\title{
Vegetation Database of Strict Forest Reserves in NW-Germany
}

\author{
Wolfgang Schmidt, Michaela Dölle \& Andreas Parth
}

\begin{abstract}
Strict forest reserves are forests without any direct anthropogenic influence such as management activities. They have been established all over Germany as well as in many other European countries to gain a substantial, representative network of protected areas. Strict forest reserves can be used as a reference for forestry and nature conservation in order to derive modern forestry practices including the main goals of sustainability and biodiversity. Besides this they offer valuable opportunities for fundamental research on forest biocoenoses. Our database comprises woodland relevés from 17 strict forest reserves (permanent plots) in Lower Saxony and Hesse (Germany). Beside single surveys, mainly repeated surveys have been conducted (time series).The database offers good opportunities to evaluate changes in time (forest dynamics, succession), impact of management activities or environmental changes on forest ecosystems. This report describes the available content in the Vegetation Database of Strict Forest Reserves in NW-Germany (GIVD ID EU-DE-016).
\end{abstract}

Keywords: Germany; permanent plot; time series.

\section{Vegetation Database of Strict Forest Reserves in NW-Germany}

Scope: The database comprises woodland relevés from 17 strict forest reserves in Lower Saxony and Hesse (Germany). The database offers good opportunities to evaluate changes in time, impact of management activities or climate change on forest ecosystems.

Status: completed and continuing Period: 1988-2011

Database manager(s): Wolfgang Schmidt (wschmid1@gwdg.de); Michaela Dölle (mdoelle@gwdg.de); Andreas Parth (aparth@gwdg.de)

Owner: University of Göttingen

Web address: http://www.uni-goettingen.de/de/67090.html

Availability: free upon request

Online upload: no

Online search: no

Database format(s): MS Access

Export format(s): [NA]

Publication: [NA]

Plot type(s): normal plots; nested plots; time series

Plot-size range: $100-400 \mathrm{~m}^{2}$

Non-overlapping plots: 3,600

Estimate of existing plots: [NA]

Completeness: [NA]

Total plot observations: 3,600

Number of sources: 1

Valid taxa: 700

Countries: DE: $100.0 \%$

Forest: [NA] — Non-forest: [NA]

Guilds: all vascular plants: $100 \%$; bryophytes (terricolous or aquatic): $80 \%$; lichens (terricolous or aquatic): $50 \%$; non-terricolous taxa (epiphytic, saxicolous, lignicolous): $20 \%$

Environmental data: altitude: $100 \%$; slope aspect: $100 \%$; slope inclination: $100 \%$; microrelief: $50 \%$; soil depth: $20 \%$; surface cover other than plants (open soil, litter, bare rock etc.): $50 \%$; soil pH: $20 \%$; other soil attributes: $100 \%$

Performance measure(s): cover: $100 \%$

Geographic localisation: GPS coordinates (precision $25 \mathrm{~m}$ or less): $100 \%$

Sampling periods: $1980-1989$ : $15.0 \%$; 1990-1999: 40.0\%; 2000-2009: 40.0\%; 2010-2019: 5.0\%

Information as of 2012-07-12; further details and future updates available from http://www.givd.info/ID/EU-DE-016

Wolfgang Schmidt (wschmid1@gwdg.de), Michaela Dölle* (mdoelle@gwdg.de), Andreas Parth (aparth@gwdg.de) Silviculture and Forest Ecology of the Temperate Zones, University of Göttingen, Büsgenweg 1, 37077 Göttingen, GERMANY

*Corresponding author 